\section{Atenção ao pré-natal na percepção das usuárias do Sistema Único de Saúde: um estudo comparativo}

\author{
Perception of prenatal care among clients \\ of the Brazilian National Health System (SUS): \\ a comparative study
}

José Mendes Ribeiro ${ }^{1}$

Nílson do Rosário Costa 1

Luiz Felipe da Silva Pinto 1,2

Pedro Luiz Barros Silva 3

\section{Introdução}

1 Departamento de Ciências Sociais, Escola Nacional de Saúde Pública, Fundação Oswaldo Cruz, Rio de Janeiro, Brasil. 2 Faculdade de Medicina, Centro de Ciências Biomédicas, Fundação Educacional Serra dos Órgãos, Teresópolis, Brasil. 3 Núcleo de Estudos de Políticas Públicas, Instituto de Economia, Universidade Estadual de Campinas, Campinas, Brasil.

Correspondência José Mendes Ribeiro Departamento de Ciências Sociais, Escola Nacional de Saúde Pública,

Fundação Oswaldo Cruz. Rua Leopoldo Bulhões 1480 Rio de Janeiro, $R J$ 21041-210, Brasil. ribeiro@ensp.fiocruz.br

\section{Abstract}

This was a comparative cross-sectional study among public prenatal care users in conventional outpatient health services and family health services, aimed at assessing perception and quality differences between the two models of health services organization according to Ministry of Health guidelines. A total of 203 pregnant women from 22 municipalities in five regions of the country were interviewed while waiting for prenatal consultation. Besides soliciting the women's opinions, we checked for possible advantages in innovative family care services in issues like access and commitment. Data revealed approval by users for key aspects related to care and consultation in both types of public facilities and suggest consistent primary care policies. Low coverage in dentistry (18.9\%), gynecological preventive tests (39.6\%), and HIV tests (52.6\%) indicates policy obstacles. Comparatively, family health services received significantly greater approval by women on issues like quality of the last visit ( $p=0.0432)$, maternity hospital access ( $p=0.0106)$, vaccination schedules ( $p=0.0023)$, drug delivery ( $p=0.0053)$, blood glucose tests ( $p=0.0309)$, nursing visit ( $p=0.0469)$, and home visits $(p<0.0001)$.

Primary Health Care; Family Health Program; Health Services
O Sistema Único de Saúde (SUS) tem sido implementado de modo incremental por meio de reformas parciais. Após mais de uma década de existência, diversas políticas foram desenvolvidas em consonância com o objetivo constitucional de prover atenção integral, hierarquizada e de caráter universal a todos os brasileiros 1 . A progressiva incorporação de novas clientelas, evidenciada pela ampliação e diversificação da oferta de serviços e redução das desigualdades regionais 2 , e a descentralização mediante fortalecimento da capacidade dos municípios 3,4, foram acompanhadas de inovações na provisão de serviços ambulatoriais e desenvolvimento de programas de saúde pública. O reordenamento do sistema revela a transferência de status decisório aos municípios, resultante de movimentos políticos setoriais que sustentaram as vantagens deste formato de descentralização 5. As inovações observadas principalmente após a emissão, pelo Ministério da Saúde (MS), da Norma Operacional Básica - SUS, em 1996 6, são sintetizadas na noção de atenção básica enquanto um conjunto de ações que envolvem ampliação da cobertura de serviços médico-assistenciais ambulatoriais; formação de equipes multidisciplinares; desenvolvimento de programas de prevenção deagravos; garantia de meios de apoio à diagnose básicos; e implementação de programas focaliza- 
dos em população de elevado risco social e epidemiológico. A literatura nacional tem ressaltado o potencial reformador da atenção básica em favor de melhores resultados sanitários e como princípio ordenador da hierarquização da oferta 7, e destaca tecnologias de ações por meio de programas de saúde coletiva como seus principais instrumentos 8 . As ações básicas enfatizam em suas rotinas o que tradicionalmente é tratado como prevenção primária 9 .

De acordo com a Norma Operacional da Assistência à Saúde - SUS 10, que induz à regionalização da oferta de serviços, são definidos sete grupos de ações estratégicas e mínimas em termos de atenção básica: controle da tuberculose; controle da hanseníase; controle da hipertensão; controle da diabetes mellitus; ações de saúde bucal; ações de saúde da criança; e ações de saúde da mulher. Os indicadores de avaliação da atenção básica preconizados monitoram o desempenho dos municípios com relação a essas ações e balizam as transferências fundo a fundo previstas com a progressão em nível de Gestão da Atenção Básica - Ampliada (GPAB-A). A avaliação do desempenho dos municípios brasileiros é feita, do ponto de vista institucional, por meio de Indicadores do Pacto da Atenção Básica. Esses indicadores, de consistência desigual, resultam de consensos estabelecidos na Comissão Intergestores Tripartite entre o MS, o Conselho Nacional de Secretários Municipais de Saúde e o Conselho Nacional de Secretários Estaduais de Saúde. Sua relevância política decorre justamente deste acordo entre os gestores do SUS com relação ao melhor modo de avaliar as redes locais de atenção à saúde e estão expressos na Portaria no 723/GM do MS 11.

Neste estudo, as Unidades Básicas de Saúde (UBS) foram selecionadas segundo os critérios do MS para definir os Postos de Saúde. Com relação aos Módulos de Saúde da Família (MSF), a seleção respeitou os critérios de habilitação do MS quando da criação do Programa Saúde da Família (PSF), em 1994. Atualmente, o padrão define cada equipe como sendo formada por um médico generalista, dois auxiliares de enfermagem e seis agentes comunitários responsáveis por cerca de 3.450 habitantes. Essas equipes atuam em módulos de portes distintos e em diferentes municípios, e podem incluir odontólogos, assistentes sociais, nutricionistas e psicólogos.

A difusão de equipes de saúde da família por todo o país resulta de experiências munici- pais exitosas, como no caso do Programa Médico de Família de Niterói (Rio de Janeiro), iniciado em 1992 12, e da forte indução pelo MS, seja por incentivos financeiros (Piso da Atenção Básica), ou por intensa emulação e divulgação de normas, objetivos e resultados. Embora a maioria destes módulos seja estabelecida em comunidades pobres, sujeitas a maiores barreiras de acesso a serviços do SUS e com baixa cobertura de planos de saúde suplementar, muitos apontam para o potencial reformador e substitutivo destes serviços com relação ao conjunto da assistência ambulatorial básica 13. Compete a esses módulos, por meio de equipes multidisciplinares de saúde, efetuar um diagnóstico social e epidemiológico de sua área de adscrição; definir microáreas de atuação; efetuar visitas domiciliares regulares com a participação de agentes comunitários; e prover atenção à saúde segundo clínicas básicas (pediatria, clínica médica, ginecologia e obstetrícia) e odontologia. Segundo características locais, a oferta de serviços é ampliada e adaptada a necessidades sociais observadas. Em termos gerais, o PSF representa um forte mecanismo de organização de acesso (porta de entrada) a serviços simples e complexos (referência), e que atualiza os conceitos tradicionais de distrito sanitário e a própria noção de hierarquização da atenção à saúde observada no texto constitucional de 1988.

Este artigo apresenta um estudo comparativo realizado junto a usuárias do pré-natal de serviços básicos do SUS, entre unidades básicas tradicionais e MSF, no sentido de identificar possíveis diferenças na percepção sobre a qualidade desses serviços nos termos das disposições normativas do programa de atenção integral à saúde da mulher, criança e adolescente.

\section{Metodologia}

A atenção ao pré-natal foi escolhida com fins comparativos para avaliar aspectos do desempenho do MSF e de outras UBS. Neste caso funciona também como um conjunto de ações que colocam em destaque processos e resultados que podem refletir o desempenho de áreas da atenção à saúde mutuamente dependentes, como a organização da porta de entrada, o sistema de agendamento de consultas, a qualidade e o acesso a serviços de laboratório e outros meios diagnósticos.

Nas pesquisas biomédica e química é comum o uso da noção de traçador, onde subs- 
tâncias, na forma de marcadores, são aplicadas de modo a colocar em evidência estruturas ou processos objetos de estudos. O seu uso em pesquisa social é menos comum, controverso e muitas vezes se confunde com a própria noção de indicador. Precursores no uso de traçadores para a avaliação da qualidade de serviços de saúde, Kessner et al. 14,15 propuseram o uso de um conjunto articulado de traçadores baseados em problemas de saúde bem definidos, pressupondo que o modo como equipes médicas administram a atenção a doenças comuns indica a qualidade geral do sistema. Para isso, apontam seis condições básicas para a seleção de traçadores: impacto funcional; diagnóstico objetivo; altas taxas de prevalência; história natural sensível à intervenção médica; conduta médica bem definida de prevenção, diagnóstico, tratamento ou reabilitação; e efeitos conhecidos de fatores não-médicos sobre o traçador. Outros autores, entretanto, utilizaram a noção de traçador, evento sentinela, rastreador ou marcador, de modo indistinto ou de acordo com a condição analisada, usando uma ou mais variáveis. Exemplos de uso de condições isoladas ou combinadas para esquemas de avaliação podem ser encontrados em áreas e desenhos metodológicos bem variados. Avila et al., 16 utilizaram a matriz estrutura/processo/resultado, elegendo a biometria hemática como rastreador da qualidade dos serviços de 52 laboratórios clínicos mexicanos. Nesse caso, embora a condição utilizada tenha sido única, cada componente do conhecido modelo de Donnabedian foi avaliado segundo conjuntos de variáveis. Hartz et al. 17 recorreram a um indicador tradicional (taxa de mortalidade infantil) e um "índice mortes evitáveis" na condição de "evento sentinela" para avaliar a qualidade da assistência à saúde em dois municípios brasileiros. No âmbito de um programa do MS (Projeto-Sentinela), Szwarcwald \& Castilho 18 estudaram uma população sentinela de gestantes para estimar, após correção dos dados disponíveis, a população brasileira de infectados pelo HIV na faixa de 15-49 anos. Em Vigilância em Saúde do Trabalhador, o recurso ao acompanhamento de evento sentinela é comum na detecção de caso-índice capaz de gerar um rol de procedimentos (busca ativa) ao longo da cadeia produtiva 19. Traçadores foram utilizados na reforma da atenção primária na Espanha por meio da identificação de hospitalizações realizadas para condições sensíveis ao atendimento ambulatorial 20. O modelo proposto por Kessner et al. 14,15 foi adaptado para o monitoramento de custos de condições traçadoras da eficiência no uso de recursos hospitalares na
Argentina 21. Um ponto comum observado nas distintas abordagens está no fato de determinadas condições ou programas serem passíveis, embora limitadamente, de sinalizar, monitorar ou evidenciar aspectos substantivos da qualidade da atenção de serviços de saúde. Funcionam como traçadores, sinalizadores, marcadores ou indicativos de condições a serem tratadas no processo decisório em políticas. Porém, se considerarmos o objetivo mais amplo de avaliar a qualidade dos serviços de saúde, nem o uso de traçadores compostos de Kessner et al. 14,15 ou usos mais pontuais seriam suficientes. Nesse caso se impõem variações de modelos de avaliação de estruturas, processos e resultados segundo a matriz conhecida de Donabedian 22. Em seu trabalho derradeiro, Donabedian 22 resume, sobre essa matriz, os componentes da qualidade a serem contemplados em um esquema adequado de avaliação de impacto sobre as condições de saúde. Este conjunto amplo reflete um modelo integrado de avaliação de políticas e inclui: eficácia (influência de inovações tecnológicas); efetividade (grau de aproximação aos aprimoramentos possíveis); eficiência (economia de custos sem prejuízo de metas); otimização (equilíbrios entre custos e ganhos em condições de saúde); aceitabilidade (conformidade a expectativas dos usuários); legitimidade (conformidade às preferências sociais e aspectos institucionais); e eqüidade (conformidade a princípios distributivos aceitos).

Neste estudo utilizamos a atenção ao prénatal para fins comparativos entre duas modalidades distintas de provisão de serviços de saúde. Destacamos ainda o potencial que este segmento possui como indicativo da qualidade dos serviços avaliados, a serem cotejados com indicadores formais de monitoramento. A escolha dos processos e atividades envolvidos na atenção ao pré-natal para fins comparativos decorre da importância que estes serviços têm no conjunto da atenção básica, tais como: oferta mínima e obrigatória; ampla distribuição nacional; exigência da disponibilidade de médico, enfermeiro, exames laboratoriais, agendamento de consultas, instalações e instrumental adequados. Além disso, a cobertura do pré-natal consiste num dos principais indicadores do Pacto da Atenção Básica do SUS. Como envolve toda a cadeia de procedimentos que os serviços devem realizar para outras ações de atenção básica, o funcionamento do pré-natal reflete aspectos da atuação de outros programas. A importância da atenção ao pré-natal como política governamental é evidente e expressa no conjunto de normas que regem a ope- 
ração do SUS. Seu impacto sobre a prevenção da prematuridade e do baixo peso ao nascer tem sido amplamente documentado, como recentemente para mães adolescentes brasileiras 23 .

O delineamento amostral foi feito com base em uma seleção intencional de 22 municípios, distribuídos por todas as grandes regiões brasileiras, baseando-se em critérios combinados de capacidade administrativa, financeira, oferta de serviços de saúde, porte populacional e região geográfica. A seleção do MSF e de UBS não teve caráter aleatório e partiu da indicação pelo gestor das unidades com melhor desempenho. A indicação foi verificada quanto às estatísticas de produção de serviços. A escolha dos melhores serviços visou a assegurar alguma homogeneidade, embora a busca por critérios mais objetivos deva ser considerada nos próximos estudos. Foram selecionadas as melhores práticas em cada município visitado, de modo a evidenciar o potencial de ambos os modelos de atenção. Após a definição desses critérios de inclusão foi selecionada uma amostra intencional de municípios. Em seguida foram selecionadas mulheres em idade fértil, segundo faixas etárias. Para a seleção da amostra foi consultado o cadastro de gestantes existente nas unidades de saúde. As gestantes foram entrevistadas na sala de espera do serviço de saúde antes do atendimento, ou em seu próprio domicílio. A opção mais adequada pela realização das entrevistas exclusivamente no próprio domicílio, de modo a minorar os efeitos do viés de cortesia, não se mostrou viável quanto aos recursos utilizados na pesquisa.

A amostra contemplou um total de 203 gestantes usuárias da rede SUS. Para permitir uma comparação inédita entre os dois tipos de serviços básicos, a amostra definida, no entanto, não representa a distribuição populacional por depender da distribuição dos MSF que existiam em menor proporção na Região Sul do que nas Regiões Nordeste e Sudeste. No entanto possui validade interna quanto ao conjunto específico de municípios selecionados. As entrevistas realizadas duraram em média vinte minutos com o uso de questionário fechado. Um estudo exploratório, de natureza transversal, foi realizado anteriormente pela equipe e envolveu dois grupos de unidades de saúde. O primeiro formado por unidades sem internação de baixa e média complexidade, que envolveu um total de 262 gestantes. O segundo grupo, composto por 203 gestantes, objeto deste artigo, foi composto por municípios que possuíam simultaneamente UBS e MSF. Esta pesquisa analisou o comportamento do grupo de 203 gestantes, distribuí- das entre um total de 104 entrevistadas (51,23\%), que estavam sendo atendidas em MSF e de 99 entrevistadas $(48,77 \%)$ em UBS. As UBS estão organizadas na forma tradicional do atendimento, desenvolvem os programas de saúde coletiva e prescindem de especialistas e serviços de referência (hoje em dia mais associados ao desenho de policlínicas). Correspondem ao porte de unidade do tipo 1 ("posto de saúde"), do Sistema de Informação Ambulatorial (SIASUS). Representam a forma de organização mais assemelhada aos MSF em termos de porte e tipo de serviços disponíveis. Algumas diferenças importantes, no entanto, devem ser observadas para fins de comparação. Os dois modelos têm suas práticas orientadas aos serviços básicos, porém o desenho organizacional é distinto: os MSF enfatizam a atenção domiciliar, as instalações físicas são próximas às moradias da população alvo e não costumam oferecer serviços de diagnóstico complementar. Os MSF dispõem de equipes de Agentes Comunitários de Saúde (ACS) que efetuam visitas domiciliares sistemáticas, ao contrário das UBS. As UBS podem dispor de laboratórios para a realização de exames simplificados e de uso extensivo (hemograma, EAS e parasitológico de fezes). Muitas UBS possuem serviços além da clínica básica, como dermatologistas, enquanto os MSF concentram os atendimentos das especialidades básicas na figura do médico generalista. Apesar dessas diferenças, que sugerem acesso mais facilitado dos usuários aos MSF e maior disponibilidade de serviços nas UBS, a comparação se justifica por dois aspectos fundamentais: os dois modelos atendem a populações alvo similares e representam a porta de entrada ao sistema para os atendimentos eletivos. Constituem formas competitivas em termos de políticas de atenção básica nos municípios. As entrevistadas foram abordadas antes de entrar na sala para a consulta do pré-natal, ou em seu próprio domicílio, seguindo-se a estratificação preestabelecida pela pesquisa, de uma mulher de até 19 anos, três com idade de 20 a 29 anos e uma com 30 anos ou mais, para cada grupo, acompanhando a distribuição das mulheres em idade fértil no Brasil. As perguntas relacionadas à percepção sobre qualidade da atenção ao prénatal foram respondidas pelas gestantes com a atribuição de notas de zero a dez e reclassificadas em sofrível, regular, bom e excelente. Para fins de comparação entre as UBS e MSF foram selecionados inicialmente os itens onde se observam diferenças estatisticamente significantes, assim consideradas as hipóteses com p-valor menor que 0,05 pelo teste de qui-quadrado de Pearson 24. Para ilustrar a comparação, em 
se tratando de pesquisa social, alguns resultados foram destacados como marginalmente significantes para p-valor menor que 0,10 quando em seu conjunto apontam para a mesma direção em itens diversos.

\section{Resultados e análise}

A aprovação pelas entrevistadas aos principais aspectos da atenção ao pré-natal que receberam nos estabelecimentos públicos foi a regra observada no estudo. Mesmo considerando que os usuários de serviços de saúde possam apresentar uma percepção mais favorável de qualidade, uma vez que tenham conseguido obter o atendimento, além da opção do estudo melhores serviços, a aprovação observada não pode ser subestimada em termos de caracterização do potencial desses serviços. Para reduzir estes efeitos, todas as gestantes foram entrevistadas antes da realização do atendimento. Além disso, pelas respostas oferecidas, não deixaram de apontar os pontos críticos do atendimento. No entanto, um controle mais eficiente deste tipo de viés deve ser feito, estimulado pelos dados obtidos, para novos estudos baseando-se em comparações com amostras de gestantes que experimentaram, por exemplo, consultas de pré-natal no setor público e no setor de saúde suplementar. Outro desenvolvimento recomendável seria a comparação entre gestantes que seguiram o acompanhamento e aquelas que abandonaram os serviços. Buscamos um detalhamento destes resultados segundo dois objetivos. Um deles diz respeito a caracterizar com maior precisão os itens responsáveis pelo desempenho observado. Isso foi alcançado pela análise deste programa com base em componentes que envolvem acesso a consultas e exames, internações, emergência, presteza nos resultados de exames, acolhimento e atitudes adotadas pelos profissionais de saúde. Outro objetivo foi comparar, no âmbito restrito daqueles municípios, os dois tipos de estabelecimentos e modelo de atenção. Diversos dirigentes do setor saúde envolvidos com o PSF, assim como documentos formais do MS, postulam a tese da maior adequação do modelo dos MSF ao atendimento primário (de porta de entrada resolutiva), por comparação ao tipo de atendimento considerado convencional prestado nas UBS. Os resultados obtidos lançam alguma luz sobre estes pressupostos. Com relação às freqüências relativas, a maioria dos dados apresentados nas tabelas mostram um desempenho superior dos MSF frente às UBS. A significância estatística destas diferenças não se manifesta, no entanto, em diversos resultados e o próprio comportamento se modifica segundo o tipo de teste utilizado. A maioria das entrevistadas (Tabela 1) considerou o atendimento ao pré-natal que vem recebendo na sua unidade de saúde de qualidade excelente $(64,0 \%)$ ou boa $(21,7 \%)$. Com relação às diferenças segundo o tipo de estabelecimento, a vantagem dos MSF para as atribuições de "excelente" $(71,2 \%$ contra $56,6 \%)$ foram marginalmente significantes $(p=0,0958)$. Adicionalmente, foi perguntado às gestantes sobre a qualidade atribuída à última consulta de pré-natal efetuada naquele estabelecimento. Os dados da Tabela 1 demonstram que a atribuição dos valores é significativamente melhor para os MSF em relação às UBS (a categoria "excelente" nos MSF é de $78,6 \%$ contra $65,0 \%$ nas UBS). Além disso, um total de $20,6 \%$ das entrevistadas das UBS consideraram esse atendimento sofrível ou regular, contra apenas $8,7 \%$ nos MSF. O estudo buscou detalhar o tipo de avaliação genérica conferida pelas entrevistadas por meio de perguntas específicas relacionadas ao tempo de espera, ao comportamento dos profissionais de saúde, à realização de exames, ao acesso aos serviços necessários e ao cumprimento dos protocolos clínicos. Com relação ao tempo de espera para o atendimento, as diferenças entre os tipos de estabelecimentos estudados não foram estatisticamente significantes, sendo relevante o fato da maioria das entrevistadas considerar que a espera não é grande para o atendimento nos estabelecimentos $(62,1 \%)$, sendo o tempo médio inferior a uma hora para $53,2 \%$ das gestantes.

Estes resultados qualificam melhor os indicativos de aprovação geral atribuída ao atendimento pré-natal. Os resultados em favor da maior presteza no atendimento observados para os MSF não foram significantes. Para caracterizar o grau de acolhimento observado nos dois modelos de atenção, as entrevistadas foram perguntadas acerca do trabalho dos profissionais de saúde. Estes valores foram considerados com relação às opiniões das gestantes quanto a atuação de médicos, enfermeiros e funcionários (definidos como agentes de portaria, atendentes, arquivistas, entre outros) para os quais emitiram notas de 0 a 10 , que posteriormente foram agrupadas entre "sofrível/regular", "bom” e "excelente". Os resultados demonstraram diferenças entre os dois tipos de estabelecimentos para médicos e funcionários. Com relação à elevada aprovação da atuação de enfermeiros, as diferenças entre os tipos de estabelecimentos não foram estatisticamente significantes. Os resultados mostrados na Ta- 
Distribuição das gestantes segundo avaliação do atendimento do pré-natal, qualidade da última consulta e tempo de espera para o atendimento. Brasil, 2000.

\begin{tabular}{|c|c|c|c|c|}
\hline Características & Respostas & MSF (\%) & UBS (\%) & Total (\%) \\
\hline Conceito geral do pré-natal & Sofrível/Regular & 12,5 & 16,2 & 14,3 \\
\hline \multirow[t]{3}{*}{$(n=203 ; p=0,0958)$} & Bom & 16,4 & 27,3 & 21,7 \\
\hline & Excelente & 71,2 & 56,6 & 64,0 \\
\hline & Total & 100,0 & 100,0 & 100,0 \\
\hline Conceito da última consulta & Sofrível/Regular & 8,7 & 20,6 & 14,5 \\
\hline \multirow[t]{3}{*}{$(n=200 ; p=0,0432)$} & Bom & 12,6 & 14,4 & 13,5 \\
\hline & Excelente & 78,6 & 65,0 & 72,0 \\
\hline & Total & 100,0 & 100,0 & 100,0 \\
\hline Existe muita espera para o atendimento? & Sim & 34,6 & 41,4 & 37,9 \\
\hline \multirow[t]{2}{*}{$(n=203 ; p=0,3183)$} & Não & 65,4 & 58,6 & 62,1 \\
\hline & Total & 100,0 & 100,0 & 100,0 \\
\hline Tempo médio de espera para & Até 1 hora & 57,7 & 48,5 & 53,2 \\
\hline \multirow[t]{3}{*}{ o atendimento $(n=203 ; p=0,1990)$} & Mais de 1 hora & 22,1 & 33,3 & 27,6 \\
\hline & Ignorado & 20,2 & 18,2 & 19,2 \\
\hline & Total & 100,0 & 100,0 & 100,0 \\
\hline
\end{tabular}

MSF = Módulos de Saúde da Família; UBS = Unidade Básica de Saúde.

bela 2 podem ser analisados pela freqüência de "excelente" atribuída pelas entrevistadas aos diferentes profissionais de saúde que atuam no estabelecimento aonde fazem seu acompanhamento de pré-natal. Esses dados revelam que, em seu conjunto, os profissionais de saúde são todos bem vistos pela maior parte de sua clientela, em todos os tipos de estabelecimentos. A avaliação positiva dos profissionais de saúde se torna evidente quando se combinam os índices de "bom" e excelente", mas se forem observados os resultados polares ("excelente" versus "sofrível/regular"), vê-se que enfermeiros e médicos se destacam na aprovação frente aos funcionários de apoio. Além disso, chama a atenção a taxa relativamente alta de entrevistadas que qualificam como "sofrível/regular" o atendimento prestado pelos médicos nas UBS $(20,4 \%)$. Não há diferenças significativas entre as aprovações a médicos e enfermeiros. A comparação do desempenho de MSF e UBS pode ser melhor observada pelo comportamento dos índices de "excelente" atribuídos pelas entrevistadas: (a) os médicos são melhor aprovados nos MSF $(75,8 \%)$ do que nas UBS $(67,7 \%)$, com diferenças apenas marginalmente significantes; (b) existe diferença marginalmente significante entre os estabelecimentos no que se refere à aprovação do trabalho dos funcionários em favor dos MSF (54,4\%) quanto às UBS
(37,8\%); (c) a desaprovação ao trabalho dos funcionários de apoio, medida pelo grau de "sofrível/regular", é maior entre as entrevistadas atendidas nas UBS $(30,6 \%)$ do que nos MSF $(19,4 \%)$, sendo as diferenças iguais marginalmente significantes. Estes dados denotam dois aspectos principais. O primeiro se refere ao fato dos profissionais envolvidos com atividades finalísticas e de formação universitária serem melhor avaliados que os de atividades meio e de formação de nível médio. Isso pode ser devido especialmente à capacidade de resolver problemas e responder ao que as entrevistadas mais se interessam, enquanto que funcionários como as atendentes são muitas vezes as responsáveis por comunicar as medidas associadas às barreiras de acesso. A própria formação cultural muito próxima que esses funcionários têm com a da clientela pode influenciar com relação aos aspectos morais da formação de status. Apesar destas ressalvas, a forte aprovação ao trabalho de médicos e enfermeiros demonstra que o apoio geral atribuído ao pré-natal é consistente entre as gestantes. $\mathrm{O}$ outro aspecto relevante diz respeito aos diversos indicativos de maior aprovação das entrevistadas ao trabalho de todos os profissionais de saúde nos MSF, refletindo maior vinculação desses profissionais às suas clientelas. A diferença de dimensões físicas e escala dos serviços não de- 
Distribuição das gestantes segundo avaliação da qualidade do atendimento e características selecionadas do pré-natal. Brasil, 2000.

\begin{tabular}{|c|c|c|c|c|}
\hline Características & Respostas & MSF (\%) & UBS (\%) & Total (\%) \\
\hline Qualidade do atendimento: & Sofrível/Regular & 9,1 & 20,4 & 14,6 \\
\hline \multirow[t]{3}{*}{ Médicos $(n=192 ; p=0,0520)$} & Bom & 15,2 & 11,8 & 13,5 \\
\hline & Excelente & 75,8 & 67,7 & 71,9 \\
\hline & Total & 100,0 & 100,0 & 100,0 \\
\hline Qualidade do atendimento: & Sofrível/Regular & 4,0 & 7,7 & 5,7 \\
\hline \multirow[t]{3}{*}{ Enfermeiros $(n=192 ; p=0,2523)$} & Bom & 17,8 & 24,2 & 20,8 \\
\hline & Excelente & 78,2 & 68,1 & 73,4 \\
\hline & Total & 100,0 & 100,0 & 100,0 \\
\hline Qualidade do atendimento: & Sofrível/Regular & 19,4 & 30,6 & 24,9 \\
\hline \multirow[t]{3}{*}{ Funcionários $(n=203 ; p=0,0730)$} & Bom & 26,2 & 31,6 & 28,9 \\
\hline & Excelente & 54,4 & 37,8 & 46,3 \\
\hline & Total & 100,0 & 100,0 & 100,0 \\
\hline Presteza na realização de exames & Sim & 75,7 & 76,3 & 76,0 \\
\hline \multirow[t]{2}{*}{$(n=200 ; p=0,9261)$} & Não & 24,3 & 23,7 & 24,0 \\
\hline & Total & 100,0 & 100,0 & 100,0 \\
\hline Rapidez no acesso aos resultados & Sim & 76,0 & 75,0 & 75,5 \\
\hline \multirow[t]{2}{*}{$(n=196 ; p=0,8707)$} & Não & 24,0 & 24,0 & 24,5 \\
\hline & Total & 100,0 & 100,0 & 100,0 \\
\hline Facilidade na marcação de consultas & Sim & 89,3 & 82,6 & 86,2 \\
\hline \multirow[t]{2}{*}{$(n=195 ; p=0,1755)$} & Não & 10,7 & 17,4 & 13,9 \\
\hline & Total & 100,0 & 100,0 & 100,0 \\
\hline Acesso de acompanhante às consultas & $\operatorname{Sim}$ & 80,2 & 68,0 & 74,1 \\
\hline \multirow[t]{3}{*}{ e exames $(n=193 ; p=0,0796)$} & Não & 9,4 & 20,6 & 15,0 \\
\hline & Ignorado & 10,4 & 11,4 & 10,9 \\
\hline & Total & 100,0 & 100,0 & 100,0 \\
\hline Conhecimento do local do parto & Sim & 56,4 & 49,0 & 52,8 \\
\hline \multirow[t]{2}{*}{$(n=199 ; p=0,2922)$} & Não & 43,6 & 51,0 & 47,2 \\
\hline & Total & 100,0 & 100,0 & 100,0 \\
\hline Facilidade de admissão em maternidade & $\operatorname{Sim}$ & 77,9 & 59,5 & 69,1 \\
\hline \multirow[t]{2}{*}{$(n=165 ; p=0,0106)$} & Não & 22,1 & 40,5 & 30,9 \\
\hline & Total & 100,0 & 100,0 & 100,0 \\
\hline Qualidade das instalações do posto & Sim & 79,6 & 71,7 & 75,7 \\
\hline \multirow[t]{2}{*}{ de saúde $(n=202 ; p=0,1907)$} & Não & 20,4 & 28,3 & 24,3 \\
\hline & Total & 100,0 & 100,0 & 100,0 \\
\hline
\end{tabular}

MSF = Módulos de Saúde da Família; UBS = Unidade Básica de Saúde. 
ve ter influenciado nas escolhas, que se referem especificamente ao modo como cada entrevistada é tratada. Para reforçar as evidências de melhor desempenho dos MSF neste item, deve ser lembrado que as UBS costumam dispor de maiores recursos laboratoriais e de manejo de urgências e emergências, que poderiam influenciar no julgamento de seus profissionais. Um fator que deve ser considerado, não avaliado no estudo, seria a influência que o padrão de formação de expectativas tem neste caso, pois existe a tendência na maioria dos municípios em montar MSF junto às comunidades mais carentes e desprovidas de serviços públicos. O maior acolhimento atribuído aos MSF pode ser explicado em função da fixação dos profissionais aos módulos devido a aspectos relacionados à gestão de pessoas (melhores salários, maior carga horária) e ao próprio modelo (uso mais intensivo de ACS e do agendamento de consultas). No entanto, outros aspectos devem ser considerados, como o fato das UBS em geral serem representadas por estabelecimentos de maior porte e maior volume de consultas e procedimentos em saúde, o que torna mais difícil a organização do acesso quando comparado aos pequenos MSF que atuam em áreas com adscrição de clientelas. Os dados da Tabela 2 destacam ainda questões acerca do acesso aos recursos laboratoriais básicos e aos seus resultados, à marcação de consultas e ao acesso de acompanhantes. Os resultados obtidos para o acesso rápido à marcação de exames e obtenção dos resultados se mostraram semelhantes nos dois tipos de estabelecimentos. Dessa forma, o fato de algumas UBS disporem de laboratórios básicos próprios parece compensado por um eficiente sistema de referência desses exames pelos MSF. Aqui também sobressaem os aspectos positivos dos serviços em seu conjunto, com $76,0 \%$ das entrevistadas afirmando que os exames pedidos na rede básica são feitos com rapidez e $75,5 \%$ que os resultados são rapidamente conhecidos. Quanto a outros elementos componentes do acesso das gestantes aos serviços, se observa que para $86,2 \%$ das entrevistadas existe facilidade para a marcação de consultas e que as diferenças entre os tipos de estabelecimentos não são significantes. Com relação ao acesso de acompanhantes às consultas, este é facilitado na avaliação de $74,1 \%$ das entrevistadas, sendo melhor o desempenho observado pelas gestantes dos MSF (80,2\%) frente às das UBS $(68,0 \%)$, com diferenças estatísticas marginalmente significantes. Outros componentes importantes da atenção ao pré-natal na rede pública básica foram estudados à luz dos resultados apresen- tados na Tabela 2. Um indicador de acesso importante diz respeito à garantia do leito para o parto. A pesquisa revela um importante obstáculo do sistema, evidenciado por $47,2 \%$ das gestantes que desconhecem o local onde será realizado o seu parto. As dificuldades são maiores para as usuárias das UBS $(51,0 \%)$ do que para as dos MSF (43,6\%), mas não são diferenças estatisticamente significantes. Para 30,9\% das gestantes, não será fácil ser admitida em uma maternidade, o que é um número muito elevado e denota barreiras de acesso. Neste item, as usuárias das UBS (40,5\%) apontam maior dificuldade no acesso ao leito em maternidade do que as dos MSF (22,1\%), mais confiantes, e as diferenças entre os grupos são significativas. Por fim, $75,7 \%$ das entrevistadas consideram boas as instalações dos estabelecimentos onde foram atendidas, com maior aprovação para os MSF $(79,6 \%)$ que para as UBS $(71,7 \%)$, porém estas diferenças entre os grupos não são significantes.

As gestantes foram perguntadas acerca do cumprimento de um rol de procedimentos básicos da atenção pré-natal preconizados pelo MS 25. As disposições deste protocolo foram redigidas em forma compreensível e apresentadas às entrevistadas, que atestaram ou não a sua realização. Deve ser considerada, no entanto, a possibilidade da gestante (apesar do questionário amigável) poder ter realizado determinado exame ou procedimento sem estar plenamente informada a respeito ou simplesmente ter se esquecido da informação recebida. Apesar disso, o instrumento se mostrou muito preciso na observação da maioria dos itens e serviu para qualificar as respostas anteriores. A lista de procedimentos que compõem as recomendações básicas do MS 25 inclui a realização de visitas domiciliares; de exame de urina verificação de gravidez; de hemograma com verificação de tipo sangüíneo; de sorologia para sífilis e AIDS; de verificação de glicemia; de exames de urina e de fezes; e de preventivo ginecológico. Além disso, o protocolo básico inclui procedimentos como adoção do cartão da gestante; recebimento de medicamentos quando necessários; verificação de cartão de vacinação; realização de consultas médicas, odontológicas e de enfermagem; mínimo de 4-6 consultas médicas de pré-natal; e agendamento de consultas. Os resultados observados para cada item são resumidos na Tabela 3.

Os dados da Tabela 3 permitem um detalhamento maior acerca dos aspectos que sustentam o tipo de aprovação conferido ao prénatal, porém revelam importantes obstáculos relacionados a provisão de determinados servi- 
Distribuição das gestantes segundo realização de itens componentes do protocolo de atenção ao pré-natal do Ministério da Saúde. Brasil, 2000.

\begin{tabular}{|c|c|c|c|c|c|c|}
\hline \multirow[t]{2}{*}{ Item } & \multicolumn{2}{|c|}{ MSF (\%) } & \multicolumn{2}{|c|}{ UBS (\%) } & \multicolumn{2}{|c|}{ Total (\%) } \\
\hline & Sim & Não & Sim & Não & Sim & Não \\
\hline Exame de urina $(n=197 ; p=0,2894)$ & 95,1 & 4,9 & 97,9 & 2,1 & 96,5 & 3,5 \\
\hline Cartão da gestante $(n=202 ; p=0,6655)$ & 96,2 & 3,8 & 94,9 & 5,1 & 95,5 & 4,5 \\
\hline Consulta médica de pré-natal $(n=201 ; p=0,5162)$ & 92,3 & 7,7 & 89,7 & 10,3 & 91,0 & 9,0 \\
\hline Exame de sangue $(n=199 ; p=0,8219)$ & 89,4 & 10,6 & 88,4 & 11,6 & 89,0 & 11,0 \\
\hline Agendamento de consultas $(n=200 ; p=0,5192)$ & 89,4 & 10,6 & 86,5 & 13,5 & 88,0 & 12,0 \\
\hline Determinação de tipo sangüíneo ( $n=184 ; p=0,1275)$ & 83,3 & 16,7 & 90,9 & 9,1 & 87,0 & 13,0 \\
\hline Vacinação atualizada ( $n=199 ; p=0,0023$ ) & 92,3 & 7,7 & 76,8 & 23,2 & 84,9 & 15,1 \\
\hline Teste imunológico de gravidez $(n=198 ; p=0,5225)$ & 79,6 & 20,4 & 83,2 & 16,8 & 81,3 & 18,7 \\
\hline Exame de sangue para sífilis (VDRL) ( $n=172 ; p=0,6530)$ & 79,6 & 20,4 & 82,3 & 17,7 & 80,8 & 19,2 \\
\hline Distribuição de medicamentos ( $n=198 ; p=0,0053$ ) & 77,9 & 22,1 & 59,6 & 40,4 & 69,2 & 30,8 \\
\hline Determinação de glicemia $(n=181 ; p=0,0309)$ & 74,0 & 26,0 & 58,8 & 41,2 & 66,9 & 33,1 \\
\hline Exame de fezes $(n=200 ; p=0,0727)$ & 70,9 & 29,1 & 58,8 & 41,2 & 65,0 & 35,0 \\
\hline Consulta de enfermagem $(n=201 ; p=0,0469)$ & 70,2 & 29,8 & 56,7 & 43,3 & 63,7 & 36,3 \\
\hline Sorologia para HIV $(n=196 ; p=0,3786)$ & 49,5 & 50,5 & 55,8 & 44,2 & 52,6 & 47,4 \\
\hline Visitas domiciliares $(n=200 ; p<0,0001)$ & 67,3 & 32,7 & 32,3 & 67,7 & 50,5 & 49,5 \\
\hline Preventivo ginecológico ( $n=197 ; p=0,9492)$ & 39,8 & 60,2 & 39,4 & 60,6 & 39,6 & 60,4 \\
\hline Consulta odontológica $(n=201 ; p=0,8115)$ & 18,3 & 81,7 & 19,6 & 80,4 & 18,9 & 81,1 \\
\hline
\end{tabular}

MSF = Módulos de Saúde da Família; UBS = Unidade Básica de Saúde.

ços. Foram incluídas no estudo gestantes usuárias que já haviam realizado ao menos uma consulta de pré-natal. Um total de $82,5 \%$ das entrevistadas já tinham realizado ao menos duas consultas. Os dados mais desfavoráveis a serem destacados são os índices elevados de gestantes que não realizaram o preventivo ginecológico $(60,4 \%)$ por fatores culturais ou não disponibilidade dos exames; testes para HIV $(47,4 \%)$ e consultas odontológicas $(81,1 \%)$. São resultados que denotam importantes obstáculos à implementação bem sucedida da política, cujo objetivo inclui a cobertura completa e integral de sua população alvo.

Os demais dados favorecem a política de pré-natal e mostram o seu enraizamento no SUS, com a existência de unidades de excelência em ambos os modelos. As diferenças entre os dois modelos não foram significativas para a maioria dos itens estudados, porém os MSF obtiveram melhores resultados e as diferenças foram significativas para a vacinação atualizada (92,3\% contra 76,8\%), distribuição de medicamentos $(77,9 \%$ contra $59,6 \%)$, determinação de glicemia $(74,0 \%$ contra $58,8 \%$ ), consulta de enfermagem $(70,2 \%$ contra $56,7 \%)$, além da esperada vantagem com relação às visitas domiciliares $(67,3 \%$ contra $32,3 \%)$.

\section{Considerações finais}

A avaliação de programas públicos em saúde na perspectiva dos usuários não é comumente observada na literatura técnica e científica brasileira. Igualmente não são conhecidos estudos comparativos abrangentes em termos de tecnologias e arranjos organizacionais de provisão da atenção básica. Entretanto, com a progressiva disseminação dos PSF e impulsionado por pesquisas desenvolvidas em programas de mestrado e doutorado e demandadas por gestores do SUS, a avaliação da atenção básica tem se tornado mais freqüente e originado artigos em periódicos nacionais. A tônica dominante desses estudos tem sido a descrição de seus fundamentos e estratégias, sua distribuição nacional e estudos de caso. Senna \& Cohen 26 apresentam estudo de caso sobre o Programa Médico de Família do Município de Niterói (Rio de Janeiro), destacando as suas peculiares e os resultados satisfatórios das inovações organizacionais, como a criação da Fundação Municipal de Saúde e seu impacto sobre a gestão de recursos humanos. Em termos de desenvolvimento de modelos ou de propostas de avaliação, o PSF tem sido alvo de interesse crescente de pesquisadores. Senna 12 destaca o debate acerca do caráter focalizado de ações como o 
PSF, que acompanha a sua implementação e considera o caráter eqüitativo desta política. Pedrosa \& Teles 27 utilizaram a técnica dos grupos focais para definir agenda de problemas entre profissionais do PSF em Teresina (Piauí). Trad \& Bastos 28 destacaram a necessidade de se incluir a análise de características culturais observadas no processo saúde/doença em modelos de avaliação do PSF. Uma avaliação do PSF enfatizando as condições de implementação política, com ênfase na caracterização dos fatores condicionantes de experiências exitosas foi realizada por Goulart 13. Na linha de avaliação de resultados de experiências exitosas, também com ênfase nos processos políticos e na implementação do programa, Escorel et al. 29 destacaram como fatores limitantes à difusão do PSF a resistência de decisores à substituição da rede básica convencional pelo novo modelo, a inadequação do modelo de financiamento do MS para os grandes centros urbanos, e sugerem para estas regiões a adaptação das unidades básicas para abrigar um conjunto limitado de equipes de saúde da família. Outros pontos destacados se referem a problemas no modo de adscrição de clientelas, lacunas no sistema de referência de usuários e a necessidade de incentivos salariais para as equipes em áreas de risco social e epidemiológico elevados.

Na literatura internacional são observados alguns estudos de satisfação ou percepção de usuárias de serviços e programas de atenção ao pré-natal. Isso inclui os países industrializados, onde a atenção à saúde é mais abrangente do que o observado em países de baixa renda, caracterizados pela focalização em programas prioritários de atenção primária, os quais têm a sua avaliação expressa em um elenco mais amplo de periódicos. Estudos de satisfação de usuárias com a atenção ao pré-natal em países industrializados costumam enfatizar o comportamento de clientelas de baixa renda. Handler et al. 30 realizaram entrevistas nos Estados Unidos com mulheres de baixa renda, comparando duas amostras com 125 beneficiárias do Medicaid (assistência à saúde voltada à população pobre) e com 275 não vinculadas ao programa e obtiveram altas taxas de aprovação, similares às descritas em nosso estudo. A destacar o fato da aprovação entre as beneficiárias do Medicaid ser comparativamente maior, apesar deste grupo registrar um número médio de consultas menor. Omar \& Schiffman 31 estudaram um grupo de sessenta mulheres de baixa renda em área rural no Estados Unidos e obtiveram igualmente elevadas taxas de satisfação. Uma amostra de 126 mulheres norte-americanas de baixa renda, com filhos menores de um ano de idade foi analisada 32 e revelou nenhum $(13,0 \%)$ ou inadequado acesso $(50,8 \%)$ aos protocolos do pré-natal, sendo a utilização desses serviços significativamente mais freqüente entre aquelas que demonstraram melhor percepção sobre a sua importância. Relatos positivos sobre a experiência do pré-natal foram predominantes, porém experiências negativas associadas a condições de acesso foram descritas, como tempo de espera para o atendimento e dificuldades com transportes. Em comum com o nosso estudo, esses foram realizados com populações pobres e as percepções favoráveis quanto aos serviços podem refletir também o modo como entendem seus direitos e estruturam suas expectativas frente aos serviços públicos.

Em nosso estudo, destacamos o caráter preliminar da avaliação dos modelos em perspectiva comparada e sob a ótica das usuárias. A generalização dos resultados deve ser considerada com as reservas apontadas quanto à amostra realizada. Por outro lado, os processos identificados junto às gestantes permitem observar aspectos importantes da atenção básica, considerando a validade interna observada no estudo. O uso da atenção ao pré-natal para fins comparativos de aspectos da atenção básica se mostrou adequado pelo conjunto de processos que envolve na provisão de serviços. A aprovação expressa pela maioria das usuárias deve ser considerada seriamente, em que pese o fato dos serviços selecionados terem atendido ao critério de maior excelência. Um possível viés pode ser apontado pelo fato das entrevistadas já serem incluídas no programa, sendo importante em estudos futuros compará-las com aquelas não incorporadas regulamente à rotina do pré-natal. Estudos fundamentados em amostras populacionais podem gerar novas evidências com relação aos dados aqui comentados. As principais conclusões, segundo a ótica das entrevistadas, podem ser resumidas em: conceito geral altamente favorável ao pré-natal, apesar do tempo médio de espera para o atendimento ser superior a uma hora ("muita espera”) assinalado por 37,9\%; elevada satisfação com o trabalho dos médicos, mais percebida no MSF ( $p=0,0520$ ), e dos enfermeiros; alta presteza na realização de exames, no acesso aos resultados e na marcação de consultas; a maioria das entrevistadas revelou expectativas favoráveis quanto ao acesso ao leito em maternidades, sendo expressiva a vantagem do MSF neste item; elevada aprovação da qualidade das instalações. O protocolo de procedimentos preconizados foi cumprido em mais de $80,0 \%$ dos casos para a maioria dos itens, e isto revela o 
enraizamento de políticas de atenção na estrutura de provisão de serviços do SUS no país. Porém, as baixas coberturas observadas em itens críticos como a determinação de glicemia, o teste de HIV-AIDS, o exame preventivo ginecológico e a realização de consultas odontológicas, mostram obstáculos importantes. As diferenças entre os dois modelos com relação ao cumprimento do protocolo do MS, quando significativas, foram sempre favoráveis aos MSF, porém chama a atenção o fato de $32,7 \%$ das entrevistadas neste tipo de estabelecimento não relatarem visitas domiciliares. Igualmente, quando os testes de significância indicaram pvalor entre 0,05-0,10, tratados como apenas marginalmente significantes, as diferenças en-

\section{Resumo}

Estudo comparativo transversal com usuárias do prénatal de serviços do SUS em unidades básicas de saúde e módulos de saúde da família (MSF), para determinar diferenças na percepção sobre a qualidade desses serviços, segundo as disposições normativas do $\mathrm{Mi}$ nistério da Saúde para esse programa. Uma amostra de 203 gestantes usuárias da rede básica pública, distribuída por 22 municípios nas cinco regiões do país foi estuda por meio de entrevistas em salas de espera dos serviços. Além de analisar as opiniões das gestantes, busca observar as possíveis vantagens das inovações em atenção familiar em termos de adesão e acesso das usuárias. A aprovação das usuárias ao atendimento recebido em ambos os modelos sugere solidez nas ações de atenção básica. A baixa cobertura de consultas odontológicas (18,9\%), preventivos ginecológicos $(39,6 \%)$ e testes de HIV (52,6\%) revelam obstáculos da política. Em termos comparativos, os MSF foram melhor avaliados significativamente quanto à qualidade da última consulta ( $p=0,0432)$, acesso a maternidades ( $p=0,0106)$, vacinação atualizada $(p=0,0023)$, distribuição de medicamentos ( $p=0,0053)$, determinação de glicemia ( $p=0,0309$ ), consulta de enfermagem ( $p=0,0469)$ e visitas domiciliares $(p<0,0001)$.

Atenção Básica; Programa Saúde da Família; Serviços de Saúde tre os dois modelos foram sempre favorável ao MSF, o que pode sugerir uma tendência geral quando vistos em conjunto os demais resultados. Os resultados relativamente favoráveis aos MSF frente às UBS apontam para o potencial do novo modelo na melhora da qualidade dos serviços públicos de saúde. Novos estudos devem considerar amostras mais representativas e controlar fatores relevantes como perfil sócio-econômico, de rede de serviços e dos órgãos gestores. Os dados revelados demostram as possibilidades reais que estudos sobre percepção e satisfação de usuários apresentam para o conhecimento das facilidades e dos obstáculos mais evidentes na busca por qualidade no âmbito do sistema público.

\section{Colaboradores}

J. M. Ribeiro, N. R. Costa e P. L. B. Silva coordenaram a pesquisa de campo que proporcionou os dados analisados no artigo. L. F. Pinto contribuiu na parte estatística da pesquisa. Todos os autores participaram do desenvolvimento da proposta metodológica, montagem do instrumento, coleta e análise de dados. 


\section{Referências}

1. Cordeiro H. Sistema Único de Saúde. Rio de Janeiro: Ayuri Editorial; 1991.

2. Ribeiro JM, Costa NR. Regionalização da assistência à saúde no Brasil: os consórcios municipais no Sistema Único de Saúde (SUS). Planej Polít Públicas 2000; 22:173-220.

3. Costa NR, Silva PLB, Ribeiro JM. A descentralização do sistema de saúde no Brasil. Revista do Serviço Público 1999; 50:33-55.

4. Costa NR, Silva PLB, Ribeiro JM. A experiência internacional de reforma do setor saúde: inovações organizacionais e de financiamento. Rev Adm Pública 2000; 34:209-27.

5. Goulart FAA. Municipalização: veredas - caminhos do movimento municipalista de saúde. Rio de Janeiro: ABRASCO; 1996.

6. Ministério da Saúde. Norma Operacional Básica SUS. Portaria no 2.203/1996, alterada pela Portaria MS/GM no 1.882 de 18/12/1997. Brasília: Gabinete Ministerial, Ministério da Saúde; 1996.

7. Mendes EV, organizador. Distrito sanitário: o processo social de mudança das práticas sanitárias do Sistema Único de Saúde. São Paulo: Editora Hucitec/Rio de Janeiro: ABRASCO; 1993.

8. Schraiber LB, organizador. Programação em saúde hoje. São Paulo: Editora Hucitec; 1990.

9. Leavell H, Clark EG. Medicina preventiva. São Paulo: McGraw-Hill do Brasil; 1976.

10. Ministério da Saúde. Norma Operacional de Assistência à Saúde - SUS. Portaria MS / GM no 95, de 26/01/2001. Brasília: Gabinete Ministerial, Ministério da Saúde; 2001.

11. Ministério da Saúde. Portaria no 723/GM de 10 de maio de 2001. Brasília: Gabinete Ministerial, Ministério da Saúde; 2001.

12. Senna MCM. Eqüidade e política de saúde: algumas reflexões sobre o Programa Saúde da Família. Cad Saúde Pública 2002; 18 Suppl:203-11.

13. Goulart FAA. Experiências de saúde da família: cada caso é um caso? [Tese de Doutorado]. Rio de Janeiro: Escola Nacional de Saúde Pública, Fundação Oswaldo Cruz; 2002.

14. Kessner DM, Kalk CE, Singer JA. Assessing health quality - the case for tracers. N Eng J Med 1973; 288:189-94.

15. Kessner DM, Kalk CE, Singer JA. Evaluación de la calidad de la salud por el método de los procesos trazadores. In: White KL, editor. Investigaciones sobre servicios de salud: una antología. Washington DC: Organización Panamericana de la Salud; 1992. p. 555-63. (Publicación Científica 534).

16. Avila L, Hernández P, Cruz A, Zurita B, Terres AM, Cruz C. Analysis of productivity, quality and cost of first grade laboratories: complete blood count. Rev Saúde Pública 1999; 33:163-70.

17. Hartz Z, Champagne F, Leal MC, Contandriopoulos AP. Mortalidade infantil "evitável” em duas cidades do Nordeste do Brasil: indicador de qualidade do sistema local de saúde. Rev Saúde Pública $1996 ; 30: 310-8$.

18. Szwarcwald CL, Castilho EA. Estimativa do número de pessoas de 15 a 49 anos infectadas pelo HIV, Brasil, 1998. Cad Saúde Pública 2000; 16 Suppl 1:135-41.
19. Vasconcellos LCF, Ribeiro FSN. Investigação epidemiológica e intervenção sanitária em saúde do trabalhador: o planejamento segundo bases operacionais. Cad Saúde Pública 1997; 13:269-75.

20. Caminal J, Sánchez E, Morales M, Peiró R, Márquez S. Investigation progress in Spain with "Ambulatory Care Sensitive Conditions". Rev Esp Salud Pública 2002; 76:189-96.

21. Villanueva H. Ongoing health management control by cost monitoring through tracer indicators. Rev Panam Salud Pública 2001; 10:405-12.

22. Donabedian A. An introduction to quality assurance in health care. New York: Oxford University Press; 2003.

23. Gama SD, Szwarcwald CL, Leal MC. Experiência de gravidez na adolescência, fatores associados e resultados perinatais entre puérperas de baixa renda. Cad Saúde Pública 2002; 18:153-61.

24. Arango HG. Bioestatística teórica e computacional. Rio de Janeiro: Guanabara Koogan; 2001.

25. Ministério da Saúde. Assistência pré-natal: normas e manuais técnicos. 3 a Ed. Brasília: Secretaria de Políticas de Saúde, Ministério da Saúde; 2000.

26. Senna MCM, Cohen MM. Modelo assistencial e estratégia saúde da família em nível local. Ciênc Saúde Coletiva 2002; 7:523-35.

27. Pedrosa JIS, Teles JBM. Consenso e diferenças em equipes do Programa de Saúde da Família. Rev Saúde Pública 2001; 35:303-11.

28. Trad LAB, Bastos ACS. O impacto sociocultural do Programa de Saúde da Família (PSF): uma proposta de avaliação. Cad Saúde Pública 1998; 14: 429-35.

29. Escorel S, Giovanella L, Mendonça MH, Magalhães R, Senna MCM. Avaliação da implementação do Programa de Saúde da Família em dez grandes centros urbanos: síntese dos principais resultados. Brasília: Secretaria de Políticas de Saúde/Ministério da Saúde; 2002.

30. Handler A, Rosenberg D, Raube K, Lions S. Satisfaction and use of prenatal care: their relationship among African-American women in a large managed care organization. Birth 2003; 30:23-30.

31. Omar MA, Schiffman RF. Satisfaction and adequacy of prenatal care utilization among rural lowincome women. Outcomes Manag Nurs Pract 2000; 4:91-6.

32. Mikhail B. Prenatal care utilization among lowincome African American women. J Community Health Nurs 2000; 17:235-46.

Recebido em $17 /$ Jun/2002

Versão final reapresentada em 08/Ago/2003

Aprovado em 23/Out/2003 